Available online on 15.06 .2020 at http://jddtonline.info
Open Access to Pharmaceutical and Medical Research
unrestricted non-commercial use, provided the original work is properly cited

Open Access

Review Article

\title{
Liquisolid Technology: Preparation, Characterization and Applications
}

\author{
Ashish Prakash Gorle*, Shubham Sunil Chopade \\ Department of Pharmaceutics, R C Patel Institute of Pharmaceutical Education and Research, Shirpur- 425405 India
}

\begin{abstract}
With the advent of high throughput screening, drugs are emerging to be more lipophilic and less hydrophilic. Liquisolid Technology aims at solubility enhancement of such entities via cosolvency concept in a relatively minimalistic setup where there is no need of sophisticated machinery and is cost effective. It involves constituting a drug into molecular dispersion via a non-volatile solvent and then transforming it into a dry looking, free flowing compressible powder. This article aims at mapping Liquisolid Technology where its preparation techniques and potential applications are reviewed. An overview of the performance of Liquisolid in areas of dissolution enhancement, zero order release, photostability enhancement, liquipellets and its role in natural product formulations is recorded for a number of drugs.
\end{abstract}

Keywords: Liquisolid, Dissolution Enhancement, Flowability, Compressibility, Cosolvency

Article Info: Received 27 March 2020; Review Completed 18 May 2020; Accepted 24 May 2020; Available online 15 June 2020

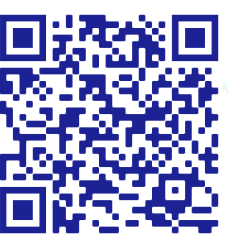

Cite this article as:

Gorle AP, Chopade SS, Liquisolid Technology: Preparation, Characterization and Applications, Journal of Drug Delivery and Therapeutics. 2020; 10(3-s):295-307 http://dx.doi.org/10.22270/jddt.v10i3-s.4067

\section{*Address for Correspondence:}

Dr. Ashish Prakash Gorle, M.Pharm., Ph.D, Associate Professor, Department of Pharmaceutics, R C Patel Institute of Pharmaceutical Education and Research, Shirpur- 425405 Dist- Dhule Maharashtra, India

\section{INTRODUCTION}

A major reason for failure of new chemical entities in clinical development is due to their poor pharmacokinetics along with toxicity or failure to prove their efficacy at minor level. It is found that most of novel drugs after lead optimization lie in the BCS class 2 or 4 which means that they are poorly soluble in water or are practically insoluble. 1 The advent of sophisticated techniques such as High Throughput Screening has led to identification of large number of chemical compounds. These compounds though lack in optimum pharmacokinetics due to their high lipophilicity, less water solubility and high molecular weight as compared to conventional drugs. Hence these properties impede the viability of the chemical compound. In the High throughput screening discovery technique, 'the rule of 5' states that poor absorption or permeation occurs when there are more than $5 \mathrm{H}$-bond donors, $10 \mathrm{H}$-bond acceptors, molecular weight is greater than 500 and the calculated $\log \mathrm{P}(\mathrm{CLOg} \mathrm{P})$ is greater than 5 (or $M \log P>4.15$ ). High throughput screening lead compounds tend to have higher molecular weight and $\log \mathrm{P}$ and lower aqueous solubility than conventional drugs. ${ }^{2}$ It is practically impossible to step back to lead compound optimization phase of the drug \& change its chemical structure just to enhance its physicochemical properties as required because of the enormous time and money already spent upon the novel agent development process. It costs anywhere near to $0.8-1.2$ billion US Dollars and 10-15 years to launch a novel drug entity in market. Hence it isn't a reasonable and viable option to send the drug back to lead optimization phase. Here formulation development plays a significant role in such cases to establish an optimum pipeline for drug delivery. ${ }^{3}$

This article is based upon one such formulation development technique viz. Liquisolid technology for improving the dissolution rate and bioavailability ${ }^{4}$ of poorly water soluble or practically insoluble drugs.

\section{THEORY OF LIQUISOLID SYSTEMS}

Liquisolid Systems are free flowing, compressible admixtures of drug solutions or suspensions. It literally consists of words 'liqui' meaning Drug Solutions or Suspensions and 'solid' meaning in a powdered form. Liquisolid Systems aims at enhancing the water solubility and in-vitro dissolution of poorly water soluble drugs belonging to BCS class 2 and 4. It utilizes Co-Solvency concept for solubility enhancement of the subjected chemical entity. It is based upon incorporation of water insoluble drug into a Non-Volatile solvent in which the respective drug is fairly soluble and converting the resultant liquid drug solution or suspension into a free flowing and readily compressible powder by using carriers with high specific surface area, porous material and high liquid absorbing capacity and nanometric (10nm-5000nm) sized coating materials showing high surface adsorption. 5 Drug Solutions 
or Suspensions mean drug of choice suspended into a suitable Non-Volatile solvent. Liquisolid systems are molecularly dispersed admixtures of such drug solutions or suspensions; hence they are dry-looking admixtures and are not exactly devoid of any moisture. Liquisolid compacts refer to compression of such admixtures into a unit dosage form. In order to achieve this, 'flowable liquid-retention potential' $(\Phi)$ \& 'compressible liquid-retention potential' $(\Psi)$ values of carrier and coating materials are screened by Liquisolid
Flowability Test (LSF) \& Liquisolid Compressibility Test (LSC) respectively. These values determine the ability \& quantity required of carrier and coat to retain its compressibility and flowability while equipped with certain amount of Liquid drug solution or suspension. ${ }^{5-6}$ Comparison between Coarse, Amorphous and Molecular Dispersions is shown in Figure 1 out of which liquisolid systems are molecular dispersions of drug in solvent.

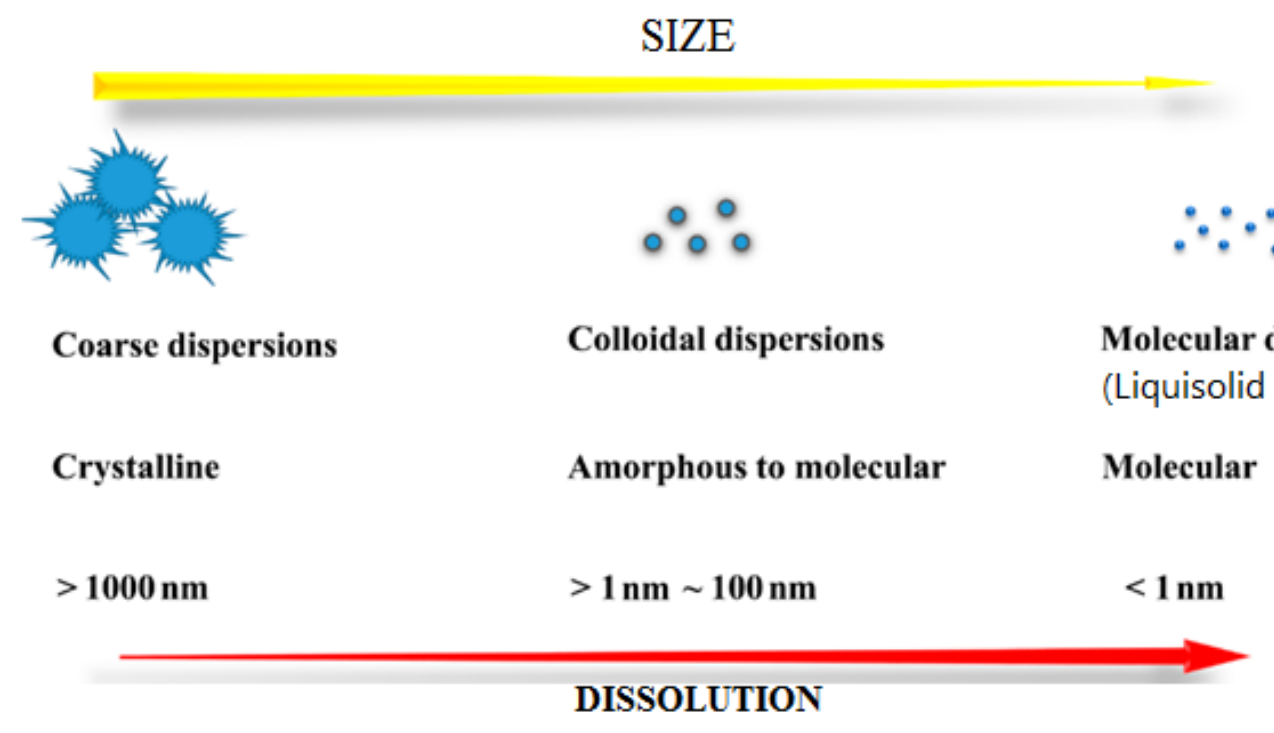

Figure 1: Difference between Coarse, Amorphous and Molecular Dispersions (Adapted from ${ }^{57}$ )

\subsection{Building Blocks for LiquiSolid Systems 5}

1. Non-Volatile Solvent- Water Miscible Solvents are selected in which the subjected water insoluble drug can be incorporated and shall be readily soluble or suspendable. The resultant Drug Solution/ Suspension should be orally safe. High Liquid Concentration has proved to enhance dissolution of the drug and low concentration is known to prolong the effect of the drug. Some Examples are Polyethylene Glycol 20011,12,17,37, Polyethylene Glycol 30024, Polyethylene Glycol 4009,20,25, Polyethylene Glycol 60017, Polysorbate 20 (Tween 20) ${ }^{33}$,Polysorbate 80 (Tween 80) 13,14,25,28,31,32,39, Propylene Glycol8,10,14,15,19,22,30, Glycerin 33 , Cremophor ${ }^{\circledR}$ EL (Polyoxyl 35 Castor Oil) ${ }^{20,23,39}$, Synperonic ${ }^{\circledR}$ PE/l61 (Poloxamer 181) 20,29,37, Labrasol25,56, Kollicoat ${ }^{\circledR}$ SR30D23,29 , Transcutol ${ }^{\circledR}$ (Diethylene Glycol monoethyl

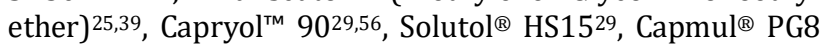
(Propylene glycol mono caprylate) ${ }^{39}$

Polysorbate 80 is such a solvent which was primarily used for retarding the drug release owning to the fact that most of the drugs have the lowest solubility in Polysorbate 80 but still higher than that of water making it an excellent retardant along with a matrix forming agent such as Eudragit $\AA$ or Hypromellulose polymers.

2. Carrier- Porous materials having high specific surface area $\&$ liquid absorption capacity can be employed as carriers which can absorb the resulting Drug Solution to convert it into a dry looking free flowing powder. Some examples are Microcrystalline Cellulose (Avicel PH 101, 102, 200)8-13,19-23,27,30-33, Amorphous Cellulose, Ethyl Cellulose ${ }^{28}$, Dibasic Calcium Phosphate, Fujicalin ${ }^{\circledR}$ (Synthetic Dibasic Calcium Phosphate Anhydrous) ${ }^{21}$, Neusilin ${ }^{\circledR}$ US2 (Synthetic Amorphous Aluminometasilicate) $21,46,56$, Eudragit ${ }^{\circledR}$ (RL,
RS) $14,28,31,33$, HPMC K4M (Hydroxy propyl ethyl cellulose) ${ }^{33}$, Starch $^{26}$, Lactose ${ }^{12,27,34}$, Mannitol ${ }^{34}$ and Florite ${ }^{\circledR}$ (Calcium Silicate) ${ }^{21}$.

Microcrystalline Cellulose is the pioneer carrier used in the Liquisolid technique as almost used in every initial research involving Liquisolid technique. Though over the time many have exploited other carriers in the search of better surface exposure of the drug to the dissolution media. Javadzadeh et al. reported comparison between different grades of Avicel ${ }^{\circledR}$ (Microcrystalline Cellulose) and its effect upon the ultimate formulation. Avicel ${ }^{\circledR} 101$ proved an increase in the flowability of the Liquisolid admixture. Avicel ${ }^{\circledR} 101$ and Avicel ${ }^{\circledR} 102$ showed an enhanced dissolution profile of the drug piroxicam in the same experiment. Avicel ${ }^{\circledR}$ $101 \&$ Avicel ${ }^{\circledR} 200$ produced hard compacts as compared to Avicel ${ }^{\circledR} 102$. Hence Javadzadeh et al. concluded that Avicel® 101 to be an optimum grade of Microcrystalline Cellulose. 16

Specific Surface area (SSA) of the powder ultimately decides the amount of wettability and surface exposure of the drug particles with the dissolution media. Hence it is essential to select a carrier which has a high Specific Surface area. Nokhodchi et al. reported surface areas of various carriers screened by BET Surface area method which are enlisted in Table 1. It was concluded that Neusilin $\AA$ US2 increases the liquid load factor of the powder blend by a factor of 7 due to its extremely high SSA. Hence Neusilin ${ }^{\circledR}$ US2 holds immense potential in not only obtaining a proficient dissolution profile but also in high dose incorporation for Liquisolid technology which is often limited by the low liquid absorption capacity of Microcrystalline Cellulose and other such carriers. ${ }^{21}$ 
Table 1: Surface areas of various powders used as carriers in Liquisolid System

\begin{tabular}{|l|l|}
\hline Carrier & BET Surface Area $\left(\mathrm{m}^{2} / \mathrm{g}\right)$ \\
\hline Fujicalin® (Synthetic Dibasic Calcium Phosphate Anhydrous) & $32 \mathrm{~m}^{2} / \mathrm{g}$ \\
\hline Avicel® PH200 (Microcrystalline Cellulose) & $1 \mathrm{~m}^{2} / \mathrm{g}$ \\
\hline Florite® (Calcium Silicate) & $142 \mathrm{~m}^{2} / \mathrm{g}$ \\
\hline Neusilin® US2 (Synthetic Amorphous Magnesium Aluminometasilicate) & $339 \mathrm{~m}^{2} / \mathrm{g}$ \\
\hline
\end{tabular}

3. Coat- Materials having very fine particle size and a highly adsorptive surface are used to adsorb any excess liquid and to seal and coat the carrier particles. A desired particle size range of these adsorptive particles is $10 \mathrm{~nm}$ $5000 \mathrm{~nm}{ }^{5}$. Amorphous Silicon Dioxide (Aerosil 200, Cab-0$\mathrm{Sil} ® \mathrm{M} 5$ ) is used widely as it fits best in the desired properties of the coat $5,8-13,19-23$, and $30-32$. Neusilin ${ }^{\circledR}$ US2 has been experimentally tried to serve a dual purpose of carrier and coat as well which resulted in a better flowability of the admixture ${ }^{21}$. Neusilin ${ }^{\circledR}$ US2 is also being used as both carrier as well as coat in the authors research on Liquisolid systems and preliminary results has shown a significant enhancement of Liquid load factor due to enhanced flowability Unpublished Data. Mesoporous Silica was screened by Chuanbin et al. which indicated a huge BET surface area of $1030 \mathrm{~m}^{2} / \mathrm{g}$ and pore size of $2.8 \mathrm{~nm}$ thus promising a high liquid adsorption capacity as compared to conventional silica 26 .

4. Adjuvants- Binders, Lubricants, Diluents, Disintegrants, Matrix forming polymers can be added as per requirement and can be blended with the parent preparation.

Super Disintegrants serve an instrumental role in Liquisolid systems to force open the tablet for disintegration due to the fact that it may be unable to do so without a disintegrant due to high amount of Non Volatile solvent concentration in the dry looking powder. Sodium Starch Glycolate is used extensively as a superdisintegrant in the Liquisolid Formulations 8-10, 13, 19, and 32. Maize Starch 20 and Crosspovidone ${ }^{39}$ were also reviewed as used in the same as Disintegrants. Vraníkova et al. reported effects of various superdisintegrants upon the liquisolid compacts of Rosuvastatin which stated Crosspovidone to be the most suitable disintegrant and a binary mixture of Crosspovidone and Sodium Starch Glycolate suitable for tablet disintegration enhancement 43-44. However, Sodium Starch Glycolate still remains the popular choice of disintegrant in most of the formulations.

Lubricants can be used as required but are a major missing from most of the Liquisolid formulations as the flowability is already achieved as desired in precompression phase of the Tablets. Though some research has reported the usage of Magnesium Carbonate 11, Magnesium Stearate 28 and Talc 28.

\subsection{Scheme of Preparation of Liquisolid Systems}

Most of the Liquisolid systems have been prepared according to the original scheme and method as reported by Spireas $\mathrm{S}$. Figure 2 explains the various steps required to formulate Liquisolid systems.
Phase 1 (Drug Solutions or Suspensions): Mix Drug + Non Volatile Solvent \& heat to $80^{\circ} \mathrm{C}-90^{\circ} \mathrm{C}$ with constant stirring until homogeneous drug solution obtained
Incorporate resultant hot liquid medication in calculated quantities of Carrier followed by 3 stages of mixing

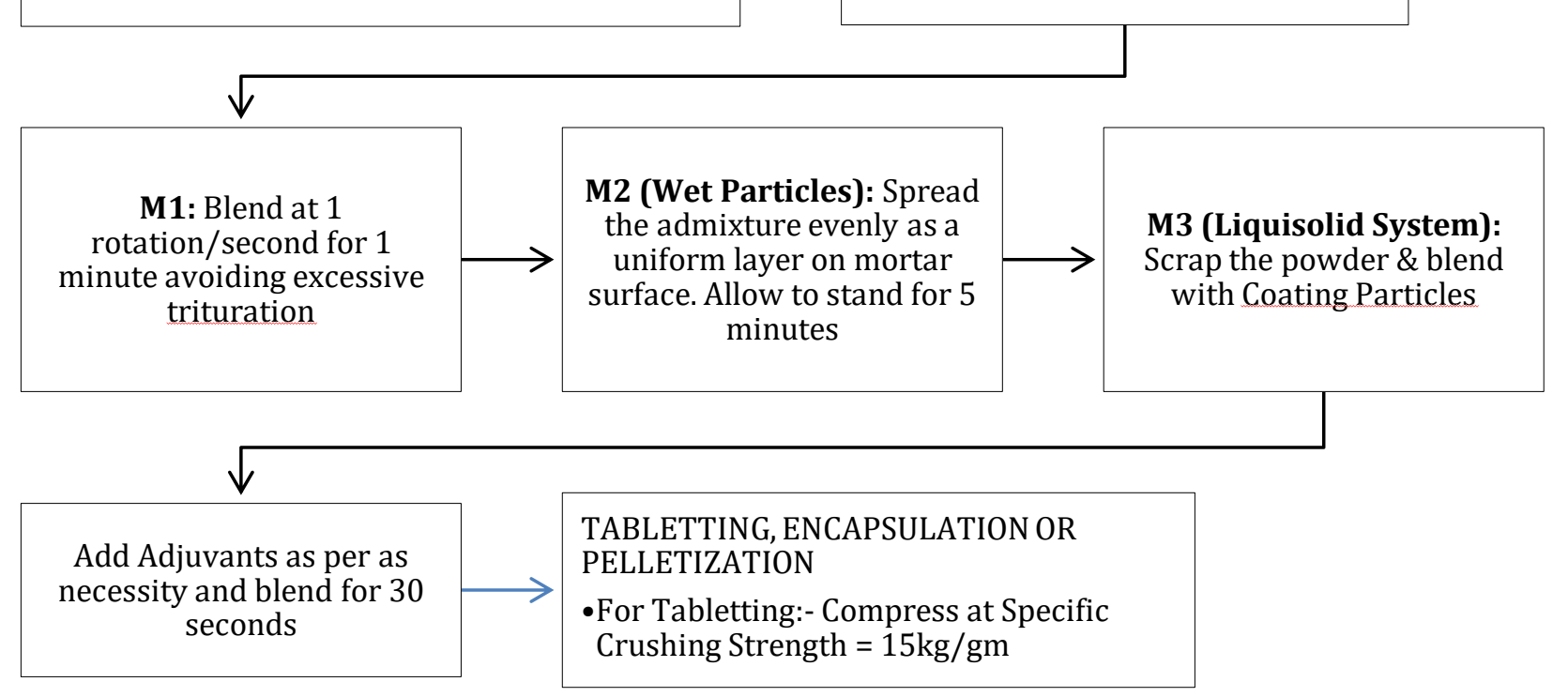

Figure 2: Scheme of Preparation of Liquisolid Systems 
Also, over the time many process developments were also recorded to achieve some enhanced results from the parent scheme. There are two such developments achieved which has reportedly enhanced the Liquid Load factor and dissolution profiles of resulting experiments.

1. Addition of binders at Phase 1 in Drug solutions or Suspensions in order to achieve an improved dissolution profile. Polyvinylpyrrolidone (PVP), Hydroxypropyle Methylcellulose (HPMC) and Polyethylene Glycol (PEG 35000) were investigated for the same where PVP as binder significantly enhanced the dissolution profile of drugs viz. Carbamazepine 12 and Glyburide 27.

2. Wet Granulation of the final Liquisolid admixture using binders viz. hydroxypropylethylcellulose 14 , acetonic solution 21 and water ${ }^{38}$. Since wet granulation involves drying of the entire powder mixture after granulation, it compromises the basic philosophy of Liquisolid admixtures to create Microsystems or molecular dispersions as such intense heat after granulation process can also evaporate the Non Volatile solvent in the liquid medication. The above experiments were successful in retarding the drug release of Propranolol Hydrochloride 14, enhancing drug release of Tocopherol Acetate 21 and Norfloxacin 38 with addition to enhancement in liquid load factor when water used as binder which is attributed to water being able to create wider spaces between the structures 38 .

\section{MATHEMATICAL MODEL EMPLOYED TO CALCULATE LIQUID LOAD FACTOR OF EXCIPIENTS 5}

These equations were originally introduced by Spireas S. in order to calculate the quantities of carrier and coat required to convert the liquid medications into a dry looking free flowing and compressible compacts. The equations are enlisted below.

$\mathrm{Lf}_{\Phi}$ (Liquid Load for acceptable Flowability) $=\Phi+\frac{\varphi}{R}(1)$

where $\Phi$ is the flowable liquid-retention potential of Carrier $\& \varphi$ of the Coating material respectively.

$\mathrm{Lf}_{\Psi}$ (Liquid Load for acceptable Compressibility) $=\Psi+\frac{\psi}{R}$ (2)

where $\Psi$ is the compressible liquid-retention potential of Carrier \& $\psi$ of the Coating material respectively.

$\mathrm{R}$ is the ratio of Carrier and Coating material to be used expressed as

$$
\mathbf{R}=\frac{\text { Carrier Weight }(Q)}{\text { Coat Weight }(q)}(3)
$$

$\mathrm{Lf}_{0}$ (Optimal Liquid Load Factor) $=\operatorname{Lf}_{\Phi}$ if $\mathrm{Lf}_{\Phi}<\operatorname{Lf} \Psi(4)$

$\mathrm{Lf}_{0}$ (Optimal Liquid Load Factor) $=\mathrm{Lf} \psi$ if $\mathrm{Lf}_{\psi}<\mathrm{Lf}_{\Phi}(5)$

Similarly weight of Carrier \& Coat can be calculated by the following expression;

$\mathrm{Lf}_{0}($ Optimal Liquid Load Factor $)=\frac{\text { Weight of Liquid Medication }(W)}{\text { Carrier Weight }(Q)}$

(6)

$$
\begin{aligned}
& \text { Hence, } \mathrm{Q}=\frac{W}{L f 0}(7) \\
& \text { Since (6); } \mathrm{q}=\frac{Q}{R}(8)
\end{aligned}
$$

\section{DETERMINATION OF $\Phi$ (FLOWABLE LIQUID RETENTION POTENTIAL) \& $\Psi$ (COMPRESSIBLE LIQUID RETENTION POTENTIAL) VALUES ${ }^{5}$}

It is evident that a powder certainly retains only a limited amount of liquid medication while maintaining an acceptable limit of flowability and compressibility. Hence, established mathematical models are used to calculate the amount of liquid the powder can be loaded with resulting into an acceptably free flowing and readily compressible 'dry looking powder'. This parameter is known as Liquid Load Factor (Lf). In order to calculate an optimal Lf, it is necessary to determine 'flowable liquid-retention potential' $(\Phi)$ \& 'compressible liquid-retention potential' $(\Psi)$ values of carrier and coating materials respectively. They are determined by Liquisolid Flowability Test (LSF) \&Liquisolid Compressibilty Test (LSC).

\subsection{Liquisolid Flowability Test (LSF)}

LSF experiment aims at screening the flow property of the powder and capping a limit to liquid load factor as required by the researcher. Hence, this technique not only serves viable for Liquisolid systems but also for regular testing of flow properties of powder with excellent reproducibility. The flow property can be tested by number of different experiments such as by a Recording Powder Flowmeter 5, Angle of Slide ${ }^{6}$ or by simple Angle of Repose experiment as per the suitability and performability of the experiment. The technique offers flexibility to decide optimum flow as required hence producing variable results for different researchers due to the independency of parameters decided by them. A schematic representation of the LSF is made in Figure 3. 
Powder systems having ratios as $R_{1}, R_{2}, R_{3} . . R_{n}$ are prepared using desired carrier and coat material. Suitable weight of each powder system was prepared sufficient for further analysis.

Liquid/Powder admixtures having ascending Lf vaules were prepared as $\mathrm{Cw}$ in quantities corresponding 10 grams of carrier and coat as per R value; For Example-

- For R=10 :- Cw1= 0.1 Lf; Since Lf= W/Q, W(Weight of Non-Volatile Solvent) = 1 gram for 10 gram of Powder admixture.

-Similary Cw2, Cw3, Cw4, Cw5 \& Cw6 having Lf values of 0.2, 0.3, 0.4, 0.5 \& 0.6 respectively were prepared for all the three Carrier:Coat ratios.

Assess the Flow rate of every admixture by any desired process established (Methods such as Angle of Repose, Angle of Slide, Powder Flow Tester experiments are recorded in literature)

Select a Lf value for each Ratio value which complies to the preselected limit of acceptable flowability

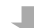

Plot the selected optimum Lf values against their respective reciprocal values of R (Carrier:Coat Ratio)

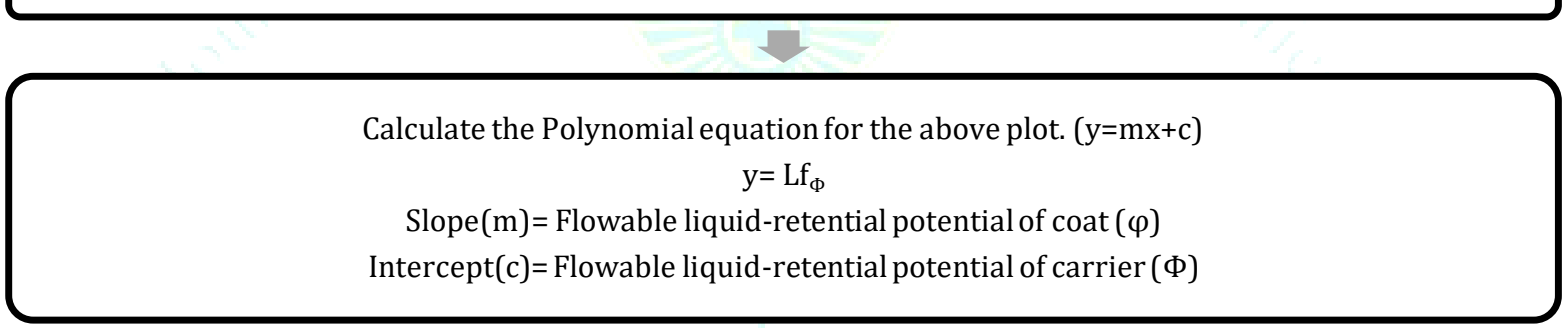

Figure 3: Scheme of Liquisolid Flowability Test (LSF)

\subsection{Liquisolid Compressibilty Test (LSC)}

LSC experiments aims at recording the maximum liquid retention possible in order to produce compacts with maximum hardness. Though if compacts are compressed at maximum hardness, they tend to create fragments or not disintegrate at all in the In-Vitro Dissolution study as recorded by the author unpublished data. Hence, in maximum researches in Liquisolid technology, it is no where reported that the $\mathrm{Lf}_{\Psi}$ was considered over $\mathrm{Lf}_{\Phi}$ due to the fact that most of the carriers can hold enormous amount of liquid if they are just to be compacted at their plateau strength and $L f \Psi$ values are always way higher than the $\mathrm{Lf}_{\Phi}$ values. Still it is a suitable way to demonstrate the capability of the powder to retain the liquid while producing acceptable compacts. If a slight modification is done for the experiment suggesting compression at desired hardness over compression at plateau strength, the technique can prove its viability and can provide with better comparisons between $\Phi \& \Psi$ values. A schematic representation of the LSC is made in Figure 4. 
Powder systems having ratios as $\mathrm{R}_{1}, \mathrm{R}_{2}, \mathrm{R}_{3} . . \mathrm{R}_{\mathrm{n}}$ are prepared using desired carrier and coat material. Suitable weight of each powder system was prepared sufficient for further analysis.

Liquid/Powder admixtures having ascending Lf vaules were prepared as $\mathrm{Cw}$ in quantities corresponding 10 grams of carrier and coat as per $\mathrm{R}$ value; For Example-

For R=10 :- Cw1= 0.1 Lf; Since Lf=W/Q, W(Weight of Non-Volatile Solvent $)=1$ gram for 10 gram of Powder admixture. Similary Cw2, Cw3, Cw4, Cw5 \& Cw6 having Lf values of $0.2,0.3,0.4,0.5$ \& 0.6 respectively were prepared for all the three Carrier:Coat ratios.

Compress tablets from each resulting admixture at plateau compression force

Calculate Pactisity $(\Omega)=$ Hardness of Tablet $\left(\mathrm{kg} / \mathrm{cm}^{2}\right) /$ Weight of Tablet $(\mathrm{gm})$

To calculate Characteristic Intrinsic Pactisity $\left(\Omega_{0}\right.$ and Sponge Index $\left(\sigma_{\mathrm{i}}\right.$ plot $\log \Omega$ on y axis versus Cw concentrations on $\mathrm{x}$ axis where $\mathrm{Cw}=$ Liquid Weight $/$ Powder Weight

Calculate the Polynomial equation for the above plot. $(y=m x+c)$

Slope $(m)=$ Modulus of Sponge Index $1 \sigma_{i 1}$

Intercept $(c)=\operatorname{Intrinsic}$ Pactisity $\left(\Omega_{0)}\right.$

Calculate $\Psi_{\text {mix }}=\left(\log \Omega_{0} \log 20\right) / \sigma_{\text {i for all the Ratio (R) values }}$

Plot the selected optimum Lf values against their respective reciprocal values of $\mathrm{R}$ (Carrier:Coat Ratio)

Calculate the Polynomial equation for the above plot. $(y=m x+c)$

$$
\mathrm{y}=\mathrm{Lf} \Psi
$$

Slope $(m)=$ Compressible liquid-retential potential of coat $(\psi)$

Intercept $(\mathrm{c})=$ Compressible liquid-retential potential of $\operatorname{carrier}(\Psi)$

Figure 4: Scheme of Liquisolid Compressibility Test (LSC)

\section{CHARACTERIZATION PARAMETERS}

\subsection{Solid State Characterization}

It involves Drug-Excipient incompatibility studies, Crystallinity determination and morphology studies of the Liquisolid admixture.

5.1.1 Fourier Transfer Infrared Spectroscopy 22, 29
It is performed for all the excipients and liquisolid mixtures to find out whether if there are any visible chemical interactions. $\mathrm{KBr}$ pellet method is widely employed for the same where substance under investigation is compressed into a pellet at the ratio of 9:1 to $99: 1$ as required and is compressed under a hydraulic press under a force of 8-10 ton. Some results from the literature are reviewed for the same. Reports extensively record presence of same peaks in the liquisolid formulation same as the other congeners inferring no possible interactions between the excipients and 
the drug. 22, 29 One report suggested the liquid vehicle forming hydrogen bonds with the drug, which majorly thus contributes to solubility enhancement which was thence attributed to the shift in peaks of the liquisolid formulation as compared to the drug. It also reported no difference between the spectra of fresh and aged liquisolid formulations confirming the stability of the formulation under stressed humidity conditions. 29

\subsubsection{X-Ray Diffractometry 12, 14, 31}

Powder X-Ray Diffraction graphs are widely studied with a perspective of comparison of drug, excipients and the resultant liquisolid formulation in order to keep a check upon the crystallinity of the formulation and its role in solubility enhancement of the drug. The samples are exposed to $\mathrm{Cu} \mathrm{K} \alpha$ radiations where they are screened for $2 \theta$ range upto $80^{\circ}$. Reports were made where no loss in crystallinity of the powder was demonstrated being attributed to particle size reduction in process for the drug and the presence of peaks resulting from microcrystalline cellulose in that study. 12, 22 Although maximum reports demonstrated an attenuated crystallinity where the flattening of the graphs were observed which was inferred by reasoning that either the drug reached an amorphous state where it is relatively more soluble, solubilization of drug in liquid vehicle or was inferred upon low drug concentration in the final formulation. 14,31

\subsubsection{Scanning Electron Microscopy 29, 31}

SEM experiment offers a great perspective upon the changes in the microenvironment and possible permutations of the formulation. Maximum studies have reported the disappearance of the crystallinity of the drug where mostly no drug crystals are observed for liquisolid formulations which is attribute to the super porous structure of the carrier that the drug which is a part of a microsystem as a molecular dispersion gets included and incorporated into the carrier matrix thus rendering the drug visually unconceivable.

\subsubsection{Differential Scanning Calorimetry 17, 29, 61}

This technique is the most favourable one where drugexcipient incompatibilities are concerned. It provides a perspective of comparision in the enthalpy changes of the drug and excipients. In a DSC sample holder 1-2 mg of sample under investigation is weighed. The sample pans are hermitically sealed and then are subjected to heat-flux DSC in the temperature region of anywhere from $30^{\circ} \mathrm{C}-300^{\circ} \mathrm{C}$, with a heating rate as required and in an atmosphere of flowing nitrogen. The following determinations are carried out: - 1 . The active drug substance and the excipients individually. 2 . Physical mixture of drug and excipients 3. Optimized liquisolid formulation. The changes in the graphs are observed to draw out inferences for any incompatibilities. Reports record no possible interactions between drugs and excipients due to their inert nature although a vast majority of results have reported a slight attenuation of the sharp peak resulting in a peak with curvature attributing to loss of crystallinity of the drug.

\subsection{Experiments involved in LSC and LSF}

\subsubsection{Operations involved in for flowability testing}

\section{(A) Carr's Index and Hausner Ratio 62}

Flowability of all liquisolid formulations and physical mixtures is assessed by determination of Carr's Index (CI) also known as percentage compressibility. The CI was calculated from the poured and tapped densities. The $\mathrm{CI}$ is calculated according to the following equation
$\mathrm{CI} \%=100\left(\frac{\text { tapped density }- \text { bulk density }}{\text { tapped density }}\right)$

Hausner Ratio $=\frac{\text { Tapped Density }}{\text { Bulk Density }}(10)$

Carr's index and Hausner ratio inferences are enlisted in Table 2

Table 2: Relationship of Carr's Index and Hausner ratio with powder flowability

\begin{tabular}{|l|l|l|}
\hline Carrr's Index & Flow property & Hausner ratio \\
\hline$\leq 10$ & Excellent & $1.00-1.11$ \\
\hline $11-15$ & Good & $1.12-1.18$ \\
\hline $16-20$ & Fair & $1.19-1.25$ \\
\hline $21-25$ & Passsable & $1.26-1.34$ \\
\hline $26-31$ & Poor & $1.35-1.45$ \\
\hline $32-37$ & Very poor & $1.46-1.59$ \\
\hline$>38$ & Very very poor & $>1.60$ \\
\hline
\end{tabular}

\section{(B) Angle of Slide 6}

10 grams of Powder of investigation is placed on the lateral end of a polished aluminium plate and then the plate is lifted on its vertical axis until the powder aggregate slides down and that at that point, theta is recorded up from the ground considered as angle of slide. 33 degrees is considered optimum and acceptable angle for the liquisolid powders.

\section{(C) Angle of Repose: 64}

For the determination of the angle of repose, a wide-opening, glass funnel was secured with its tip at a pre-determined height above a sheet of paper placed on a horizontal surface. Twenty five grams of powder was allowed to slide slowly through the tip of the funnel resulting in the formation of the conical pile of powder. The angle of repose was calculated using the following relationship:

$\tan \theta=\frac{h}{r}$

Where $\alpha$ is the angle of repose, and $h$ and $r$ are the height and radius of the base of the conical pile, respectively. A value of $\alpha<30^{\circ}$ indicates 'excellent' flow whereas $\alpha>56^{\circ}$ indicates very poor flow. The intermediate scale indicates good ( $\alpha$ between 31 and $35^{\circ}$ ), fair ( $\alpha$ between 36 and $40^{\circ}$ ), passable which may hang up ( $\alpha$ between 41 and $45^{\circ}$ ), and poor which must be agitated or vibrated ( $\alpha$ between 46 and $\left.55^{\circ}\right)$

\subsubsection{Operations involved in for compressibility testing 55}

\section{(A) Walker Compressibility Coefficient, W 65}

The value of Walker compressibility coefficient, $\mathrm{W}$, is determined by linear regression analysis by plotting the relative volume, $\mathrm{V}$, of the compacts as a function of log compression pressure, $\log \sigma_{\mathrm{c}}$, according to Walker equation (Table 3). The value of compressibility coefficient, $\mathrm{W}$, was determined from the slope of the straight line. The relative volume, $\mathrm{V}$, of powder blend is calculated as the inverse of the relative density of the compact.

(B) Kawakita Parameters, a and 1/b 66

The values of $\mathrm{P} / \mathrm{C}$ are plotted as a function of compression pressure, $\mathrm{P}$, according to the Kawakita equation (Table 3 ). The value of Kawakita parameter, a, is obtained from the reciprocal of the slope of the straight line and the parameter, 
$1 / b$, was calculated from the value of $y$-intercept, $1 / a b$, and parameter, a.

\section{(C) Mean Yield Pressure, $\mathrm{P}_{\mathrm{y}} 67$}

The value of mean yield pressure, $\mathrm{P}_{\mathrm{y}}$, is calculated from the inverse of the slope value, $k$, obtained from the linear segment of the plot of compact density vs. compression pressure data according to Heckel equation (Table 3).

(D) Compactibility parameter, $\sigma_{0}$, and Bonding capacity, $\mathrm{b} 68$

The values of compactibility parameter, $\sigma 0$, and bonding capacity, b, are determined using Ryshkewitch-Duckworth equation (Table 3 ). The nonlinear regression analysis of the plot of tensile strength, $\sigma_{\mathrm{t}}$, as a function of compact porosity, $\epsilon$, yields intercept, $\sigma_{0}$, which is tensile strength of the material at zero porosity and is indicative of compactibility of the powder material; the slope value of the plot represents the bonding capacity, b, of the powder material under increasing pressure.

(E) Compactibility parameter, $\sigma_{\text {tmax }}$, and Compression susceptibility, $\gamma^{69}$

By plotting tensile strength of the compacts, $\sigma_{t}$, and the product of compression pressure, $\sigma_{c}$, and relative density, $\rho_{r}$, of the compacts according to Leuenberger equation (Table 3 ), the values of compactibility parameter or maximum tensile strength of the compacts, $\sigma_{\text {tmax }}$, and compression susceptibility, $\gamma$, are computed using nonlinear regression analysis.

Table 3: Mathematical models for compressibility and compactibility screening of liquisolid systems.

\begin{tabular}{|c|c|c|c|}
\hline Powder Property & Model & Equation & Constants \\
\hline \multirow[t]{3}{*}{ Compressibility } & Walker & $\begin{array}{l}V=-W \log \\
\sigma_{c}+V_{r}\end{array}$ & $\begin{array}{l}\text { W is Walker compressibility coefficient, and } V_{\mathrm{r}} \text { is the initial relative } \\
\text { volume of powder material at } 1 \mathrm{MPa} \text { pressure } 65\end{array}$ \\
\hline & Kawakita & $\frac{P}{C}=\frac{P}{a}+\frac{1}{a b}$ & $\begin{array}{l}\text { The value of Kawakita parameter, a, represents the engineering } \\
\text { strain or the degree of volume reduction at maximum pressure } \\
\left(\mathrm{C}_{\max }\right) \text {, and the value of } 1 / \mathrm{b} \text { represents the pressure to achieve an } \\
\text { engineering strain of a/ } 2 \text { which can be correlated to the plasticity } \\
\text { or as deformation capacity of the material } 66 .\end{array}$ \\
\hline & Heckel & $\begin{array}{l}\ln \left(\frac{1}{1-\rho r}\right)=\mathrm{k} \\
\sigma_{\mathrm{c}}+\mathrm{A}\end{array}$ & $\begin{array}{l}1 / \mathrm{k} \text { gives a material-dependent constant known as mean yield } \\
\text { pressure, } P_{y} \text {, which is the ability of the powder material to deform } \\
\text { plastically under pressure } 67 \text {. }\end{array}$ \\
\hline \multirow[t]{2}{*}{ Compactibility } & $\begin{array}{l}\text { Ryshkew } \\
\text { itch- } \\
\text { Duckwor } \\
\text { th }\end{array}$ & $\sigma_{\mathrm{t}}=\sigma_{0} \mathrm{e}^{-\mathrm{b} \varepsilon}$ & $\begin{array}{l}\sigma_{\mathrm{t}} \text { is the tensile strength of compact, } \sigma_{0} \text { is the tensile strength of } \\
\text { compact at zero porosity, } \mathrm{b} \text { is bonding capacity of powder particles } \\
\text { under increasing pressure, and } \varepsilon \text { is porosity of the compact } 68 \text {. }\end{array}$ \\
\hline & $\begin{array}{l}\text { Leunberge } \\
\mathrm{r}\end{array}$ & $\begin{array}{l}\sigma_{\mathrm{t}}=\sigma_{\mathrm{tmax}}(1- \\
\left.\mathrm{e}^{-\gamma \sigma c \rho \mathrm{r}}\right)\end{array}$ & $\begin{array}{l}\sigma_{\text {tmax }} \text { is the maximum tensile strength of compact at relative density } \\
\left(\rho_{\mathrm{r}} 1\right) \text {, and } \gamma \text { is compression susceptibility that characterizes the } \\
\text { deformation behavior of material } 69 \text {. }\end{array}$ \\
\hline
\end{tabular}

\section{LITERATURE REVIEW}

\subsection{In Dissolution enhancement}

Liquisolid Systems are widely entrenched as models of dissolution enhancement of poorly water soluble drugs. Since its inception in 1998, many researchers have repeatedly validated Liquisolid technology with respect to its solubility enhancement capacities and potential to be formulated as unit dosage forms. For majority of the studies, it has been probated that Liquisolid compacts enhance dissolution by Co-Solvency mechanism which results into formation of a molecular dispersion of the poorly water soluble drug material into a water miscible non volatile vehicle which thus improves the wettability of the drug. This improvisation of wettability can also be attributed to the carrier molecules carrying these molecular dispersions to the dissolution media which provides a high surface contact of drug with dissolution media owning to the high specific surface area (SSA) of Liquisolid carriers. Poorly water soluble drugs belonging to BCS Class $2 \& 4$ viz. Prednisolone 8, Methyclothiazide 9, Piroxicam 13, Indomethacin 15, Famotidine 18, Carbamazepine 19, 26, Valsartan 22, Candesartan Cilexitil 32, Simvastatin 52 and Nimodipine 56 demonstrated an excellent improvisation of dissolution profile with statistical significance as compared to their commercially available counterparts and directly compressed tablets. Even if all of them do not share a common non volatile solvent used for formulating liquid medications, a common thread is that every research listed above uses the solvent in which the drug shows maximum solubility. Hence it can be concluded that choice of solvent cannot possibly affect the dissolution mechanism if the choice of solvent is based upon maximum solubility of drug into various solutions. 8, 9, 13,15, 18, 19, 22, 26, 32, 52, 56

Similarly many other results were published with varied diaspora of findings which can lead to further prospective developments into the technique. Spireas S. et al. reported that drug release rates were independent of the volume used to simulate the GI fluid in In-Vitro dissolution testing in case of drug Hydrocortisone. Thus this study proved Liquisolid systems advantageous in actual variable physiological conditions where GI fluid constantly varies. This study also hints towards zero order release achievable by Liquisolid technique which was further confirmed by others. 10 Javadzadeh et al. reported usage of Polyvinylpyrrolidone (PVP) as an additive in drug solutions which improved dissolution of Carbamazepine 12 whilst the same was confirmed by Singh et al. using PVP in drug solution of Glyburide 26. Cremophor® EL (polyethoxylated castor oil) was identified as a potentially viable solvent when it was formulated as Liquisolid systems having Naproxen 20 and Griseofluvin 23 as model drugs. They showed significant 
improvement in dissolution profile as compared to their commercial tablets. Similarly Labrasol ${ }^{\circledR}$ was confirmed by Eztimibe Liquisolid systems 25 and Synperonic $®$ PE/L-61 by Norfloxacin 37 and Spirolactone 29 where in case of Spirolactone Liquisolid systems, it additionally identified Capryol $^{\mathrm{TM}} 90$ \& Synperonic $\AA$ PE/L-61: Solutol ${ }^{\circledR}$ HS 15 as promising liquid vehicles.

Results such as no statistical significance between commercial or conventional form and Liquisolid form of Famotidine was recorded although a visible difference in release pattern was prevalent due to rapid dissolution of the Liquisolid Compact as compared to the conventional tablet 18. In case of Norfloxacin as reported by Suliman et al. the drug showed an unprecedentedly high dissolution with Synperonic ${ }^{\circledR}$ PE/L-61 as compared to Polyethylene Glycol 200 contradicting the fact that Norfloxacin had a relatively low solubility in Synperonic $₫$ PE/L-61 as compared to Polyethylene Glycol 200. ${ }^{37}$ Further research upon the same was elucidated by the author addressing the problem of low liquid load factor and inability of high dose incorporation. Hence the researcher tried to solve the same by Wet Granulation technique with the carrier material before it was subjected to exposure to hot liquid medication. The process allegedly resulted into creation of wider spaces inside the carrier structure ultimately enhancing the liquid load factor from 0.2 to 0.4 in case of Polyethylene Glycol 200 and 0.14 to 0.29 in case of Synperonic $\AA$ PE/L-61. There were no significant differences in dissolution profiles when compared with the liquisolid formulations without granulation hence ultimately proving the efficiency of the process. ${ }^{38}$

Neusilin ${ }^{\circledR}$ US2 was screened as carrier which bore astounding results as compared to the conventional carriers used previously for the same. Jadhav et al. reported a phenomenal enhancement of the liquid load factor to 1.25 with Neusilin® US2 hence opening varied possibilities of high dose incorporation which was an initial drawback for the Liquisolid technique. Progesterone as a model drug was successfully formulated using Neusilin ${ }^{\circledR}$ US2 as carrier and showed improved dissolution as compared to conventional forms of the same. ${ }^{46}$ Same was confirmed by Hentzschel et al. for Griseofluvin. ${ }^{24}$

In one of its kind, a different perspective upon the Liquisolid systems was perceived by Lu M. et al. where they employed Raman Spectroscopy in screening of Tadalafil Liquisolid systems which indicated loss in crystallinity demonstrated by alteration of intensity as compared to pure drug. It gives us an insight into amorphous conversions of drug molecules resulting into better aqueous solubility which was also confirmed by X-Ray diffraction studies. This study also proved the homogeneous distribution of the drug across the Liquisolid system demonstrated by even tone of the light intensity in the graph. ${ }^{41} \mathrm{~A}$ different study employed mixed Co-Solvency technique where they recorded that upon using 35\% Sodium Caprylate, 5\% Sodium Benzoate and 5\% Niacinamide with Rifabutin followed by formulation of liquid medication by suspending it into Propylene Glycol, it showed a twofold dissolution enhancement as compared to in-house developed fast release capsules. It surprisingly showed no loss in crystallinity by X-Ray diffraction studies. 51

\subsection{In-Vivo and Ex-Vivo performance of Liquisolid systems}

Liquisolid system has also proved itself in its In-Vivo performance enhancing oral bioavailability of poorly water soluble drugs. It was first confirmed by Khaled et al. where they recorded $\sim 15 \%$ in-vivo oral bioavailability enhancement of Hydrochlorthiazide in Beagle dogs also entrusting a greater absorption rate as compared to the conventional tablets. It also identified its dissolution rate enhancement. This particular feature can be attributed to better wettability of drug due to liquisolid formulation. ${ }^{11}$ Badawy et al. identified and evaluated Mosapride Citrate's simulated bioequivalence performance by using biorelevant media viz. $0.1 \mathrm{~N}$ Hydrochloric Acid, A hypoacidic stomach model (pH 5 Acetate buffer), and a transfer model simulating intestinal transfer from pylorus. This model demonstrated successful prediction of in vivo behavior of the drug which was further confirmed by Human volunteer's in-vivo study which indicated oral bioavailability of drug at Relative bioavailability of $121.2 \%$ as compared to commercial tablets thus establishing the biorelevant model reliable for adaption in further research. 34 Oral Bioavailability enhancement was similarly recorded for Gliclazide 35 , Risperidone ${ }^{45}$ and Pioglitazone ${ }^{53}$ liquisolid formulations via in-vivo experiments. Permeation enhancement was demonstrated by experiments conducted by Sanka et al. where they not only recorded enhanced in-vitro dissolution for Clonazepam, but also confirmed the potential of permeation enhancement by liquisolid system of Clonazepam which demonstrated enhanced permeation in diffusion studies. Although clonazepam is highly lipophilic, hence permeation enhancement can also be attributed to its natural property and also to the fact that since it was a exvivo experiment, higher concentration in diffusion media may have resulted into enhanced permeation due to solubility enhancement of clonazepam. ${ }^{36}$ Similarly the same inferences were drawn out for Raloxifene $\mathrm{HCl}$ which demonstrated 2 fold ex-vivo intestinal permeation enhancement. 39

\subsection{In Sustained Release Formulations}

Although liquisolid systems are majorly substantiated for dissolution enhancement purposes, many have tried identifying their suitability for retarding the release of some drugs. Out of which the very first cofirmation was laid by Javadzadeh et al. where they recorded an optimal retardation and better dissolution profiles of Propranolol Hydrochloride by using Eudragit ${ }^{\circledR}$ RL as carrier. 14 Nokhodchi et al. reported same for Theophylline using Hydroxypropylethylcellulose additionally with Eudragit $₫$ RL \& RS with its synergistic action retarded the drug release more than without HPMC due to matrix formation. 17 Trimetazidine Hydrochloride was formulated as sustained release tablets using Tween $\AA 80$ as Non Volatile solvent and Eudragit $\AA$ L100 as matrix forming agent. It confirmed a prolonged release of the drug. ${ }^{28}$ Venlafaxine Hydrochloride as a model drug was formulated using Tween $\AA 80$ as Non Volatile solvent and Eudragit ${ }^{\circledR}$ RS \& HPMC respectively as matrix forming agent which showed sustained release of 9 hours in case of Eudragit ${ }^{\circledR}$ RS and 12 hours for HPMC respectively. It can thus be understood that HPMC highly retards the drug release due to its hydrophobic nature. ${ }^{31}$

\subsection{Photostability}

Number of drugs upon exposure to light loses their potency due to photodegradation which also may result into toxic rendering of drugs. Hence, applicability of this technique was exploited to conclude whether if it can produce better results as compared to conventional formulations. Khames et al. evaluated the photostability of Amlodipine liquisolid tablets and conventional tablets were tested according to ICH- Q1B guidelines. Titanium Dioxide and Silicone Dioxide were used as coating materials in ratios of $1: 1$ and 2:1. The tablets were irradiated with varied light sources as specified in the ICH guidelines for eight hours. The liquisolid tablet of 
Amlodipine not only resulted in enhanced dissolution but also proved the applicability of the technique in protection of photosensitive drugs where drug retention in liquisolid tablet was recorded at $97.37 \%$ as compared to $73.8 \%$ of the conventional tablet of Amlodipine after irradiation $(\mathrm{P}<0.05)$. Also an inverse proportionality was recorded between photoprotective effects of liquisolid systems with respect to the carrier: coat ratio (R). Though a fact cannot be overlooked that only one study in this domain has been conducted in the 22 year old history of Liquisolid Systems thus owning a future prospective for study of its photoprotective effects. 30

\subsection{Zero Order Release}

Although Propranolol Hydrochloride is a BCS Class 1 drug, it differs if the conventional matrix type approach is compared with liquisolid approach in which both relatively sustain the release of the drug but liquisolid technology has a plus point of achieving a near zero order release which is much acceptable and desirable than the first order release of the conventional matrix type tablet approach. ${ }^{14}$ The same near zero order release was also achieved by Syed et al. for Trimetazidine Dihydrochloride. 28 Thus these results establish the application of the liquisolid technique for achieving zero order release.

\subsection{Usage of Natural polymers and in Herbal Formulations}

Application of liquisolid technology upon herbal origin entities was first pioneered by Sharma et al. 2016 where they formulated poorly soluble curcumin which also has a very low oral bioavailability. Curcumin was selected as a model drug, Polysorbate 80 was selected as non volatile solvent, Avicel® PH 102 as carrier and Aerosil ${ }^{\circledR}$ as coat respectively. The results indicated a greater enhancement in magnitude of drug release of liquisolid tablet as compared to conventional tablets at any given time point. Liquisolid tablets of curcumin achieved $100 \%$ release within two hours as compared to $89.2 \%$ of conventional tablets without liquisolid microsystem. Ex-vivo permeation studies were carried out on gastric mucosa of New Zeland rabbits where also enhanced permeation was observed. In-vitro cytotoxicity was screened in n87 cancer cells to find out the differences between activity of the new formulation. A slight lower cytotoxicity was observed for liquisolid tablet as compared to pure curcumin which can be attributed to presence of excipients. In-vivo studies conducted in ravvits indicated an amazing 15.4 folds enhancement of $c_{\max }$ and 8.28 folds enhancement of absorption rate constant $\left(\mathrm{k}_{\mathrm{a}}\right)$ and 18.6 folds enhancement of relative bioavailability of curcumin which can be attributed to the enhanced release of the drug due to co-solvency by liquisolid technology. 40

Oregano essential oil via its powder extract was formulated into liquisolid and was directly compared to a freeze dried counterpart of it. Rosmarinic acid and Cavacrol are its active constituents which are poorly soluble due to which it has the potential to act as a model drug for liquisolid technology. $70 \%$ Ethanol with 30\% Glycerol was used as cosolvent due to extraction constraints of the drug and Neusilin ${ }^{\circledR}$ US2 as carrier was selected for liquisolid formulation. Ethanol was evaporated in due process. For freeze dried particles of the model drug, oregano powder extract was suspended in ethanol and then ethanol removal was induced by Rotary evaporator further hydration of film and freeze drying the mixture at $-80^{\circ} \mathrm{C}$ overnight. Quantitative analysis was performed using UPLC. Dissolution enhancement was achieved by 2.2-2.8 times for Rosmarinic acid and 2.9 times for Cavacrol thus establishing the technique's applicability for herbal extracts containing essential oils. ${ }^{47}$ Similarly
Curcuma comosa, a thai herb having estrogenic like action was also constituted as liquisolid by using Propylene Glycol as cosolvent, microcrystalline cellulose as carrier and colloidal silica as coat. Polyvinylpyrollidone was used as an additive. The herb's active constituent was extracted by maceration. Dissolution enhancement was clearly recorded. This study introduced the potential of liquisolid tablets for formulation of viscous and oleoresins like natural crude extracts. 48

Pathak et al. 2019 presented a path breaking study upon potential usage of many natural origin gums as matrices and carriers for liquisolid technology by modifying them as needed. This study indicated usage of modified polysaccharides as potential carriers which was achieved by modifying the natural gums viz. Tamarind kernel powder, Guar gum and Locust bean gum. Modification was achieved by a process where the raw powders were first suspended in water and were allowed to swell overnight which was further dried via evaporation leaving a highly porous structure. These treated powders were co-ground with mannitol (1:1) in order to achieve a better flowability of the gums. These modified polysaccharides were screened for particle size by Diffuse reflectance spectroscopy and for Specific surface area via Gas adsorption technique further equating with BET equation. Results were observed which proved the treated modified powders as optimum fot liquisolid systems as particle size was exponentially reduced for modified powders and SSA increasing by $359.3 \%$, $425.53 \%$ and $384.39 \%$ respectively for modified Guar gum, Tamarind kernel powder and Locust bean gum. Also even size distribution was observed inferred by lowered span values of modified powders. To screen these new powders Paclitaxel was used as a model drug to be incorporated into them with Solutol HS15® as cosolvent. Dissolution enhancement was observed as compared to conventionally compressed tablet. Ex-vivo permeation enhancement of $61.59 \%$ was recorded for optimized liquisolid batch. A remarkable enhancement of $\mathrm{IC}_{50}$ cytotoxic potential of $<20$ $\mathrm{mmol} / \mathrm{L}$ was observed for NCL-N87 along with high cell death values in early and late apoptosis compared to conventional tablet $(\mathrm{P}<0.05)$. Oral bioavailability enhancement of 5.43 times was observed. This can also be attributed to the fact that due to enhanced release, much of drug was available for the system to be absorbed. Thus this study proved the applicability of the natural polymers for liquisolid technology. However a clear distinction was not made in the study indicating the merits of these modified polysaccharides upon the conventionally employed carriers for liquisolid technology. 54

\subsection{Liquipellet! Is it really novel?}

A study titled liquipellet was initially pioneered by Pezzini et al. 2016 where they introduced a new concept of liquipellet which addresses the loopholes of poor flowability and compactibility of liquisolid admixtures. It was developed primarily to enhance the liquid load factor along with the flow and compressible properties of the liquisolid microsystems. This study aimed at investigating liquipellet's feasibility for felodipine. Cremophor ${ }^{\circledR}$ EL was used as cosolvent, microcrystalline cellulose as carrier and crosspovidone as coat. It created soft and porous structures which were further pelletized via Extrusion-Spheronization process where the admixture was wetted with $1 \%$ crosspovidone $\mathrm{w} / \mathrm{v}$ in water and was further dried in fluidized bed. It was compared with conventional pellets without liquisolid microenvironment resulting into enhanced dissolution. Although no comparison was made with liquisolid tablets to prove the viability of liquipellet. Also since they claimed usage of crosspovidone for first time 
as a coat, it was not compared with any of the conventionally used coating materials such as colloidal silica. 42 Later studies expanded and cleared upon the scope of liquipellet where Nokhodchi et al. 2019 addressed high dose incorporation and poor flowability and compressibility as inspiration for liquipellet where they formulated liquipellet using Naproxen as model drug. Tween ${ }^{\circledR} 80$ and Kolliphor ${ }^{\circledR}$ EL were used as solvents, Avicel ${ }^{\circledR}$ PH101 as carrier and Aerosil@ 300 as coat. Significant dissolution enhancement was observed along with a high Lf of 1 . Due to enhanced flowability resulted by pelletization, Lf increases significantly allowing high dose incorporation. 60 Pezzini et al. reported ritonavir as model drug for liquipellet where significant Lf enhancement was achieved at 1.52 for $100 \mathrm{mg}$ dose of drug which is rare for incorporation in conventional liquisolid admixtures. Dissolution efficiency (DE60min) enhancement was observed at 1.7 times and 8.29 times as compared to Ritonavir polymorph 1 \& 2 respectively. Excellent flow properties and narrow size distribution was achieved due to pelletization. ${ }^{58}$ Although this technique was purported as novel in the titles of these studies, the only novelty which stands here is the problem solving mechanism of this study related to conventional liquisolid admixtures which have deficiencies in flowability and compressibility. Liquipellet formulation overcomes this problem by pelletization of the conventional liquisolid admixtures. Hence, it is relatively nefarious to brand the technique as completely novel as it is mostly based upon the original work of Spiridon Spireas of methods of preparation of liquisolid systems. ${ }^{5}$

\subsection{Stability Considerations}

A major concern about liquisolid technology revoloves around presence of solvents in a molecular dispersion form which invites prospectus to microbial growth, contamination or even degradation of product which can result into loss of activity. Hence, stability studies were conducted through various studies where all of them reported no loss in drug activity or form after prolonged periods of time. Trimetazidine dihydrochloride liquisolid tablets were subjected to accelereated stability studies for 6 months at $40 \pm 2^{\circ} \mathrm{C} / 75 \pm 25 \% \mathrm{RH}$ where the drug content was as $96.75 \pm 0.12 \%$ at the end of $12 \mathrm{~h}$ of dissolution testing. The percentage of TZH released from the optimized formulation before storage was $81.56 \pm 0.49 \%$, where post stability study release was $82.45 \pm 0.44 \%$. Hence, it can be inferred that there was no significant loss in activity of the drug. ${ }^{28}$ Similarly Mosapride citrate liquisolid tablets were evaluated for accelerated stability studies at $40 \pm 2^{\circ} \mathrm{C} / 75 \pm$ $25 \%$ RH according to ICH guidelines 2003 for 3 and 6 months respectively. Similarity factor for drug dissolution profiles compared to fresh tablets was 84.812 and 68.07 after 3 and 6 months respectively. 34 Venlafaxine $\mathrm{HCl}$ liquisolid tablets were subjected to aging studies by storing nine tablets of various formulations at $30 \circ \mathrm{C} / 65 \% \mathrm{RH}$ and $40 \circ \mathrm{C} / 75 \% \mathrm{RH}$ for 3 months. After this time period, samples were tested for their crushing strength and dissolution compared with the freshly tested tablets. The crushing strength of liquisolid formulations was affected by the storage at high humid conditions. Liquisolid formulations containing Avicel as a carrier material have shown a decrease in their hardness, but they still have acceptable hardness and can withstand handling. This decrease may be due to moisture sorption into cellulose structure which increase molecular mobility of MCC and reduce intermolecular attraction forces. Liquisolid formulation containing Eudragit RS PO as a carrier has shown an increase in its hardness. The glass transition temperature of Eudragit RS PO is $50^{\circ} \mathrm{C}$. The presence of Tween 80 may reduce this temperature by its plasticizing effect; hence at high temperature of storage, the glassy state of polymer may be affected, which alters the physical properties of tablets. Effect on hardness was most visible at 40 o $\mathrm{C} / 75 \% \mathrm{RH}$. Whilst no significant difference on release rate of drug were visible for the same. 31

\section{CONCLUSION}

Liquisolid system provides a sleek and simple interface for formulation development for poorly aqueous soluble drugs. It is cost effective as compared to parallel solublility enhancement techniques. It has extensively proved itself feasible in dissolution enhancement, achieving zero order release for sustained release formulations, maintaining photostability of drugs, protection of natural agents from degradation and maintaining stability despite its internal humid conditions. Future prospectus lies in enhancing the poor compressibility and flowability of liquisolid admixtures along with exploration of other possible applications of the technique.

\section{REFERENCES}

1. Stenberg P, Bergström CAS, Luthman K, Artursson P. Theoretical Predictions of Drug Absorption in Drug Discovery and Development. Clinical Pharmacokinets. 2012; 41:877899

2. Lipinski CA, Lombardo F, Dominy BW, Feeney PJ. Experimental and computational approaches to estimate solubility and permeability in drug discovery and development settings. Advanced drug delivery reviews. 2012 Sep 13; 64:4-17

3. Ghadi R, Dand N. BCS class IV drugs: Highly notorious candidates for formulation development. Journal of Controlled Release. 2017 Feb 28; 248:71-95

4. Toutain PL, Bousquet-Mélou A. Bioavailability and its assessment. Journal of veterinary pharmacology and therapeutics. 2004 Dec; 27(6):455-66

5. Spireas S. Liquisolid systems and methods of preparing same. US6423339B1; 2002

6. Spireas SS, Jarowski CI, Rohera BD. Powdered solution technology: principles and mechanism. Pharm Res 1992; 9:1351-8

7. Grover R, Spireas S, Lau-Cam C. Development of a simple spectrophotometric method for propylene glycol detection in tablets. J Pharm Biomed Anal 1998; 16:931-8

8. Spireas S, Sadu S. Enhancement of prednisolone dissolution properties using liquisolid compacts. Int $\mathrm{J}$ Pharm 1998;166:1771-88

9. Spireas S, Wang T, Grover R. Effect of powder substrate on the dissolution properties of methyclothiazide liquisolid compacts. Drug Dev Ind Pharm 1999; 25:163-8

10. Spireas S, Sadu S, Grover R. Invitro release evaluation of hydrocortisone liquisolid tablets. J Pharm Sci 1998; 87:867-72

11. Khaled KA, Asiri YA, El-SayedYM. In vivo evaluation of hydrochlorothiazide liquisolid tablets in beagle dogs.Int J Pharm 2001; 222:1-6

12. Javadzadeh Y, Jafari-Navimipour B, Nokhodchi A. Liquisolid technique for dissolution rate enhancement of a high dose water-insoluble drug (carbamazepine). Int J Pharm 2007; 341:26-34

13. Javadzadeh Y, Siahi MR, AsnaashariS,et al. An investigation of physicochemical properties of piroxicam liquisolid compacts. Pharm Dev Technol 2007; 12:337-43

14. Javadzadeh Y, Musaalrezaei L, Nokhodchi A. Liquisolid technique asa new approach to sustain propranolol hydrochloride release from tablet matrices. Int J Pharm2008; 362:102-8

15. Javadzadeh Y, Siahi MR, Asnaashari S, et al. Liquisolid technique as a tool for enhancement of poorly water-soluble drugs and evaluation oftheir physicochemical properties. Acta Pharm 2007; 57:99-109

16. Javadzadeh Y, Shariati H, Movahhed-Danesh E, et al. Effect of some commercial grades ofmicrocrystalline cellulose on flowability, compressibility, and dissolution profile of 
piroxicam liquisolid compacts. Drug Dev Ind Pharm 2009; 35:243-51

17. Nokhodchi A, Aliakbar R, Desai S, Javadzadeh Y. Liquisolid compacts: the effect of cosolvent and HPMC on theophylline release. ColloidsSurfB Biointerfaces 2010; 79:262-9

18. Fahmy RH, Kassem MA.Enhancement of famotidine dissolution rate through liquisolid tablets formulation: in vitro and in vivo evaluation. EurJPharm Biopharm 2008; 69:993-1003

19. Tayel SA, Soliman II, Louis D. Improvement of dissolution properties of Carbamazepinethrough application of the liquisolid tablet technique. EurJ Pharm Biopharm 2008; 69:342-347

20. Tiong N, Elkordy AA. Effects of liquisolid formulations on dissolution of naproxen. Eur J Pharm Biopharm 2009; 73:373-84

21. Hentzschel CM, Sakmann A, Leopold CS. Suitability of various tableting excipients as carriersforliquisolid systems.Drug Development and Industrial Pharmacy. 2011; 37(10):12001207

22. Chella N, Shastri N, Tadikonda RR. Use of the liquisolid compact technique for improvement of the dissolution rate of valsartan. Acta Pharmaceutica Sinica B. 2012 Oct 1; 2(5):5028.

23. Elkordy AA, Essa EA, Dhuppad S, Jammigumpula P. Liquisolid technique to enhance and to sustain griseofulvin dissolution: Effect of choice of non-volatile liquid vehicles. International Journal of Pharmaceutics. 2012 Sep 15; 434(1-2):122-32.

24. Hentzschel CM, Alnaief M, Smirnova I, Sakmann A, Leopold CS. Enhancement of griseofulvin release from liquisolid compacts. European Journal of Pharmaceutics and Biopharmaceutics. 2012 Jan 1; 80(1):130-5.

25. Khanfar M, Sheikh Salem M, Hawari R. Formulation factors affecting the release of ezetimibe from different liquisolid compacts. Pharmaceutical development and technology. 2013 Apr 1; 18(2):417-27.

26. Chen B, Wang Z, Quan G, Peng X, Pan X, Wang R, Xu Y, Li G, Wu $C$. In vitro and in vivo evaluation of ordered mesoporous silica as a novel adsorbent in liquisolid formulation. International journal of nanomedicine. 2012; 7:199.

27. Singh SK, Srinivasan KK, Gowthamarajan K, Prakash D, Gaikwad NB, Singare DS. Influence of formulation parameters on dissolution rate enhancement of glyburide using liquisolid technique. Drug development and industrial pharmacy. 2012 Aug 1; 38(8):961-70.

28. Pavani E, Noman S, Syed IA. Liquisolid technique based sustained release tablet of trimetazidine dihydrochloride. Drug Invention Today. 2013 Dec 1; 5(4):302-10.

29. Elkordy AA, Tan XN, Essa EA. Spironolactone release from liquisolid formulations prepared with Capryol $^{\mathrm{TM}} 90$, Solutol® HS-15 and Kollicoat ${ }^{\circledR}$ SR 30 D as non-volatile liquid vehicles. European journal of pharmaceutics and biopharmaceutics. 2013 Feb 1; 83(2):203-23.

30. Khames A. Liquisolid technique: a promising alternative to conventional coating for improvement of drug photostability in solid dosage forms. Expert opinion on drug delivery. 2013 Oct $1 ; 10(10): 1335-43$.

31. Khanfar M, Sheikh Salem M, Kaddour F. Preparation of sustained-release dosage form of Venlafaxine $\mathrm{HCl}$ using liquisolid technique. Pharmaceutical development and technology. 2014 Feb 1; 19(1):103-15.

32. Sayyad FJ, Tulsankar SL, Kolap UB. Design and development of liquisolid compact of candesartan cilexetil to enhance dissolution. Journal of pharmacy research. 2013 May 1; 7(5):381-8.

33. Wang L, Feng R, Gao J, Xi Y, Huang G. Generic sustained release tablets of trimetazidine hydrochloride. Expert Opin Emerg Drugs. 2010; 15:283-98.

34. Badawy MA, Kamel AO, Sammour OA. Use of biorelevant media for assessment of a poorly soluble weakly basic drug in the form of liquisolid compacts: in vitro and in vivo study. Drug delivery. 2016 Mar 23; 23(3):808-17.

35. Mahajan HS, Patil SK, Nerkar PP. Liquisolid Compact of Gliclazide for Enhanced Dissolution and Oral Bioavailability. IJNDD Oct-Dec 2014; 6(4): 314-320.

36. Sanka K, Poienti S, Mohd AB, Diwan PV. Improved oral delivery of clonazepam through liquisolid powder compact formulations: in-vitro and ex-vivo characterization. Powder technology. 2014 Apr 1; 256:336-44.

37. Suliman AS, Anderson RJ, Elkordy AA. Norfloxacin as a model hydrophobic drug with unique release from liquisolid formulations prepared with PEG200 and Synperonic PE/L-61 non-volatile liquid vehicles. Powder technology. 2014 May 1; 257:156-67.

38. Suliman AS, Anderson RJ, Elkordy AA. Preparation of novel optimum liquisolid compacts via incorporating water granulation process to enhance the powder characterizations and dissolution behavior of a poorly soluble drug: Norfloxacin. Powder Technology. 2019 Sep 1;354:259-70.

39. Komala DR, Janga KY, Jukanti R, Bandari S, Vijayagopal M. Competence of raloxifene hydrochloride loaded liquisolid compacts for improved dissolution and intestinal permeation. Journal of Drug Delivery Science and Technology. 2015 Dec 1; 30:232-41.

40. Sharma V, Pathak K. Effect of hydrogen bond formation/replacement on solubility characteristics, gastric permeation and pharmacokinetics of curcumin by application of powder solution technology. Acta Pharmaceutica Sinica B. 2016 Nov 1; 6(6):600-13.

41. Lu M, Xing H, Yang T, Yu J, Yang Z, Sun Y, Ding P. Dissolution enhancement of tadalafil by liquisolid technique. Pharmaceutical development and technology. 2017 Jan 2; 22(1):77-89.

42. Pezzini BR, Beringhs AO, Ferraz HG, Silva MA, Stulzer HK, Sonaglio D. Liquisolid technology applied to pellets: Evaluation of the feasibility and dissolution performance using felodipine as a model drug. Chemical Engineering Research and Design. 2016 Jun 1; 110:62-9.

43. Vraníková B, Pavloková S, Gajdziok J. Experimental design for determination of effects of superdisintegrant combinations on liquisolid system properties. Journal of pharmaceutical sciences. 2017 Mar 1; 106(3):817-25.

44. Vraníková B, Gajdziok J, Doležel P. The effect of superdisintegrants on the properties and dissolution profiles of liquisolid tablets containing rosuvastatin. Pharmaceutical development and technology. 2017 Feb 17; 22(2):138-47.

45. Khames A. Investigation of the effect of solubility increase at the main absorption site on bioavailability of BCS class II drug (risperidone) using liquisolid technique. Drug delivery. 2017 Jan $1 ; 24(1): 328-38$.

46. Jadhav NR, Irny PV, Patil US. Solid state behavior of progesterone and its release from Neusilin US2 based liquisolid compacts. Journal of Drug Delivery Science and Technology. 2017 Apr 1; 38:97-106.

47. Baranauskaite J, Kopustinskiene DM, Masteikova R, Gajdziok J, Baranauskas A, Bernatoniene J. Effect of liquid vehicles on the enhancement of rosmarinic acid and carvacrol release from oregano extract liquisolid compacts. Colloids and Surfaces A: Physicochemical and Engineering Aspects. 2018 Feb 20; 539:280-90.

48. Jaipakdee N, Limpongsa E, Sripanidkulchai BO, Piyachaturawat P. Preparation of Curcuma comosa tablets using liquisolid techniques: in vitro and in vivo evaluation. International journal of pharmaceutics. 2018 Dec 20; 553(12):157-68.

49. Lu M, Xing H, Jiang J, Chen X, Yang T, Wang D, Ding P. Liquisolid technique and its applications in pharmaceutics. asian journal of pharmaceutical sciences. 2017 Mar 1; 12(2):115-23.

50. Nokhodchi A, Hentzschel CM, Leopold CS. Drug release from liquisolid systems: speed it up, slow it down. Expert opinion on drug delivery. 2011 Feb 1; 8(2):191-205.

51. Barua D, Indurkhya A, Maheshwari RK, Patel PK. Formulation of Rifabutin Liquisolid system using mixed solvency concept and their evaluation. International Journal of Research in Pharmacy and Pharmaceutical Sciences. 2019 Feb 11; 4(2): 712.

52. Anzilaggo D, Beringhs AO, Pezzini BR, Sonaglio D, Stulzer HK. Liquisolid systems: understanding the impact of drug state (solution or dispersion), nonvolatile solvent and coating material on simvastatin apparent aqueous solubility and flowability. Colloids and Surfaces B: Biointerfaces. 2019 Mar 1; 175:36-43.

53. Bonthagarala B, Dasari V, Kotra V, Swain S, Beg S. Quality-byDesign based development and characterization of pioglitazone loaded liquisolid compact tablets with improved 
biopharmaceutical attributes. Journal of Drug Delivery Science and Technology. 2019 Jun 1; 51:345-55.

54. Sharma V, Pathak K. Liquisolid system of paclitaxel using modified polysaccharides: In vitro cytotoxicity, apoptosis study, cell cycle analysis, in vitro mitochondrial membrane potential assessment, and pharmacokinetics. International journal of biological macromolecules. 2019 Sep 15; 137:2031.

55. Mamidi HK, Mishra SM, Rohera BD. Determination of maximum flowable liquid-loading potential of Neusilin $®$ US2 and investigation of compressibility and compactibility of its liquisolid blends with PEG (400). Journal of Drug Delivery Science and Technology. 2019 Dec 1; 54:101285.

56. Prajapat MD, Butani SB, Gohel MC. Liquisolid: A promising technique to improve dissolution efficiency and bioavailability of poorly water soluble nimodipine. Journal of Drug Delivery Science and Technology. 2019 Oct 1; 53:101135.

57. Zhang $\mathrm{X}$, Xing $\mathrm{H}$, Zhao $\mathrm{Y}, \mathrm{Ma}$ Z. Pharmaceutical dispersion techniques for dissolution and bioavailability enhancement of poorly water-soluble drugs. Pharmaceutics. 2018 Sep; 10(3):74.

58. De Espíndola B, Beringhs AO, Sonaglio D, Stulzer HK, Silva MA, Ferraz HG, Pezzini BR. Liquisolid pellets: A pharmaceutical technology strategy to improve the dissolution rate of ritonavir. Saudi Pharmaceutical Journal. 2019 Jul 1; 27(5):702-12.

59. Lam M, Ghafourian T, Nokhodchi A. Optimising the release rate of naproxen liqui-pellet: a new technology for emerging novel oral dosage form. Drug Delivery and Translational Research. 2020 Feb 1; 10(1):43-58.
60. Lam M, Ghafourian T, Nokhodchi A. Liqui-pellet: the emerging next-generation oral dosage form which stems from liquisolid concept in combination with pelletization technology. AAPS PharmSciTech. 2019 Aug 1; 20(6):231.

61. Van Dooren AA. Design for drug-excipient interaction studies. Drug Development and Industrial Pharmacy. 1983 Jan 1; 9(12):43-55.

62. Carr RL, Carr RL, Carr RI, Carr R. Evaluating flow properties of solids. Chem. Eng. 1965; 72:163-168.

63. Saw HY, Davies CE, Paterson AH, Jones J. Correlation between powder flow properties measured by shear testing and Hausner ratio.

64. Powder Flow. The United States Pharmacopeia 30/National Formulary 25 (USP/NF). United States Pharmacopeial Convention, Rockville, MD, USA; 2007:1174

65. Walker EE. The properties of powders. Part VI. The compressibility of powders. Transactions of the Faraday Society. 1923; 19(July):73-82.

66. Nordström J, Welch K, Frenning G, Alderborn G. On the physical interpretation of the Kawakita and Adams parameters derived from confined compression of granular solids. Powder Technology. 2008 Mar 10; 182(3):424-35.

67. Sonnergaard JM. A critical evaluation of the Heckel equation. International journal of pharmaceutics. 1999 Dec 20; 193(1):63-71.

68. Duckworth W. Discussion of ryshkewitch paper by winston duckworth. J. Am. Ceram. Soc.. 1953; 36:68.

69. Leuenberger $\mathrm{H}$. The compressibility and compactibility of powder systems. International Journal of Pharmaceutics. 1982 Sep 1; 12(1):41-55. 